Department of

Orthopaedics,

Sahlgrenska University

Hospital/Östra,

SE-416-85 Göteborg,

Sweden

$S$ Brandsson

E Faxén

B I Eriksson

L Swärd

O Lundin

J Karlsson

Correspondence to:

Dr S Brandsson.

Accepted for publication 23 July 1998

\title{
Reconstruction of the anterior cruciate ligament: comparison of outside-in and all-inside techniques
}

\author{
Sveinbjörn Brandsson, Eva Faxén, Bengt I Eriksson, Leif Swärd, Olof Lundin,Jon Karlsson
}

\begin{abstract}
The aim of this prospective study was to compare two arthroscopic techniques for reconstructing the anterior cruciate ligament, the "outside-in" (two incisions) and the "all-inside" (one incision) techniques. The results obtained for 30 patients operated on using the "outside-in" technique (group I) were compared with those for 29 patients operated on using the "all-inside" technique (group II). Before surgery, there were no significant differences between the groups in terms of Lysholm score, Tegner activity level, patellofemoral pain score, or knee laxity. Both groups displayed significant improvements in Lysholm score after 24 months, from 69 (16) to 91 (9) in group I and from 70 (17) to 90 (15) in group II (means (SD)). There were also significant improvements in patellofemoral pain scores in both groups, from 13 (6) to 18 (5) in group I and from 14 (6) to 18 (4) in group II after $\mathbf{2 4}$ months. No difference was found between the groups in knee stability at the 24 month follow up. The IKDC score was identical in both groups at follow up. The operation took significantly longer for patients in group I (mean 94 (15)) than for those in group II (mean $86(20))(p=0.03)$. The mean sick leave was $7.7(6.2)$ weeks in group I and 12.3 (9.7) weeks in group II (p $=0.026$ ), indicating that there may be a higher morbidity associated with the "allinside" technique. It can be concluded that there were no significant differences between the two different techniques in terms of functional results, knee laxity, or postoperative complications. The results were satisfactory and the outcome was similar in both treatment groups.

(Br f Sports Med 1999;33:42-45)
\end{abstract}

Keywords: anterior cruciate ligament; surgical reconstruction techniques; knee; arthroscopy

Despite the many technical advances in anterior cruciate ligament (ACL) reconstruction surgery in the last decade, the success of the procedure has remained stable over the last quarter of a century and there is no clear superiority of one procedure over another. ${ }^{1}$

In 1963 Jones $^{2}$ described a method using the central third of the patellar tendon as an ACL graft. He used open surgery with suture fixation of the graft proximally and continuity distally. Since then, major developments in surgery for ACL insufficiency have taken place utilising minimally invasive techniques. Today the free bone-patellar tendon-bone graft is considered to be the "gold standard" in ACL surgery. ${ }^{3}$

The introduction of the arthroscopically assisted technique by Dandy et $a \hat{l}$ in 1982 has made the procedure less invasive. The "outsidein" technique (rear entry) and "all-inside" technique (endoscopic) are probably the methods that are most frequently used at present. Few studies have, however, compared these techniques in a prospective manner. ${ }^{6-8}$ Harner et al found no significant difference in the results of these techniques in terms of stability and function. In their study, 27/50 patients were operated on using allografts. Aglietti et $a l^{6}$ and Santori et $a l^{8}$ found no difference in the outcome of these two methods in their prospective studies.

The one incision technique is favourable in that it avoids a further incision on the lateral aspect of the thigh.

The aim of this study was to compare the functional results and objective outcomes after using the "outside-in" and "all-inside" techniques in a prospective controlled study.

\section{Patients and methods}

STUDY DESIGN

The study was prospective and all patients were followed up by an independent observer, who was not involved in the surgical procedure. The patients were allocated randomly to the two groups. Patients scheduled for ACL reconstruction were allocated to either the "outsidein" or "all-inside" technique. The randomisation procedure was as follows. Patients were allocated to the two treatment groups using closed envelopes. In order to ensure that the surgical procedures were as uniform as possible, the patients were operated on in two consecutive series, starting with the "outside-in" technique (pre-randomisation). All patients were informed about this randomisation procedure before surgery and only those who accepted the randomisation were included in the study. The study was approved by the ethics committee of Gothenburg University. Thirty patients were operated on at one centre using the "outside-in" technique (group I) and 29 patients were operated on using the 
"all-inside" technique (group II) by one of three experienced knee surgeons who were well acquainted with both methods. The experience of surgeons operating on both groups was identical. The interval between the index injury and the reconstruction was 3-92 months, with no difference between the groups. The indication for entry into the study was a symptomatic ACL instability. All patients had undergone supervised rehabilitation without success before deciding on ACL reconstruction.

SURGICAL TECHNIQUE

The patients were operated on under general anaesthesia. In both groups, the bone-patellar tendon-bone graft was harvested through a 6-7 $\mathrm{cm}$ long anterior central skin incision. The width of the graft was $8-10 \mathrm{~mm}$ depending on the width of the patellar tendon. Meniscal pathology was dealt with. To avoid graft impingement, a notch plasty was always performed. The tibial tunnel was drilled with an $8-10 \mathrm{~mm}$ drill. In patients in group I, a 6-7 $\mathrm{cm}$ long lateral thigh incision was made over the iliotibial band. With the knee flexed at $90^{\circ}$, a guidewire was advanced into the knee and passed in either the 11 or 1 o' clock position into the posterior intercondylar notch. The guidewire was overdrilled with an $8-9 \mathrm{~mm}$ drill using the "outside-in" technique. The tibial tunnel was drilled in a similar fashion in both groups. The graft was placed in the correct position under arthroscopic control, and fixation was performed with interference screws at both ends. In the "all-inside" group, the femoral guide was passed through the tibial tunnel and a $9 \mathrm{~mm}$ femoral tunnel was drilled in either the 11 or 1 o' clock position. The fixation technique was the same in the two groups, except that the femoral screw was inserted from the rear entry in group I and from the inside in group II.

\section{POSTOPERATIVE REHABILITATION}

All the patients underwent identical postoperative rehabilitation. During the first two postoperative weeks, a rehabilitation brace was used $\left(10-90^{\circ}\right)$ and full weight bearing was permitted immediately after the operation. After three weeks, an active range of motion exercises was started and full range of motion was permitted. Training with a stationary bicycle and swimming with flutter kicks started as soon as the wound had healed. After five weeks, increased functional and strength training was permitted. After 16 weeks, jogging at a slow tempo was begun and was increased over the next few weeks in terms of speed, as well as speed variations and distance. Sports specific training was begun after 18 weeks. After about 20 weeks, team training was started. A return to sports activity was permitted after 25 weeks, if the patients had regained full power and functional stability in the operated leg.

ASSESSMENT OF CLINICAL OUTCOME

Stability measurements using a KT-1000 arthrometer (MEDmetric, San Diego, California, USA), Lysholm score, ${ }^{8 a}$ Tegner activity level, ${ }^{8 \mathrm{~b}}$ patellofemoral pain score, and one leg hop quotient were made before the operation
Table 1 Lysholm score before and three, 12, and 24 months after anterior cruciate ligament reconstruction surgery. Values are mean (SD)

\begin{tabular}{llll}
\hline & Group I & Group II & p Value \\
\hline Before surgery & $69(16)$ & $70(17)$ & 0.84 \\
3 months & $90(70)$ & $87(12)$ & 0.25 \\
12 months & $94(8)$ & $88(15)$ & 0.06 \\
24 months & $91(9)$ & $90(15)$ & 0.57 \\
\hline
\end{tabular}

Table 2 Tegner activity level. Values are mean (SD)

\begin{tabular}{llll}
\hline & Group I & Group II & p Value \\
\hline Pre-injury & $6.8(1.8)$ & $7.0(2.1)$ & 0.74 \\
12 months & $5.4(1.9)$ & $4.4(2.2)$ & 0.06 \\
24 months & $5.7(2.0)$ & $4.9(2.3)$ & 0.16 \\
\hline
\end{tabular}

and at three, 12, and 24 months afterwards. The IKDC score $^{8 c}$ was registered at the final two year follow up.

\section{STATISTICS}

A Mann-Whitney non-parametric U test was used in the comparisons of the two treatment groups. Wilcoxon's signed rank test was used in the comparisons of intraindividual function before and after surgery. $p<0.05$ was regarded as statistically significant. The results are given as mean (SD) unless median (range) is indicated.

\section{Results}

No significant differences in preoperative variables were found between the groups. The male:female ratio was $19: 11$ in group I and 20:9 in group II. The median age was 27 (range 17-42) years in group I and 27 (range 15-45) years in group II.

The mean operation time was 94 (15) minutes in group I compared with 86 (20) minutes in group II $(p=0.03)$. The mean hospital stay was 2.9 (1.2) (range 1.5-7.0) days in group I and 2.6 (1.2) days (range 1.0-7.0) in group II $(\mathrm{p}=0.34)$. The mean sick leave was 7.7 (6.2) weeks in group I and 12.3 (9.7) weeks in group II $(\mathrm{p}=0.026)$.

\section{KNEE FUNCTION}

Before surgery, the mean Lysholm score was 69 (16) in group I and 70 (17) in group II, and afterwards it had improved in both groups at the three, 12 and 24 month follow ups. At the 24 month follow up, it was 91 (9) in group I and 90 (15) in group II ( $\mathrm{p}=0.57)$ (table 1). There was no difference between the two groups either before or after the operation.

The pre-injury Tegner activity level was identical in both groups. At the 24 month follow up, the activity level was significantly lower in both groups than before the injury but there was no difference between the groups (table 2).

Group I had significantly higher values for the oneleg hop quotient before the operation but, during the follow up period, no significant difference was found between the groups (table 3 ).

\section{LAXITY}

No significant difference was found in the KT-1000 values between the groups before surgery. After surgery, the KT-1000 values improved significantly in both groups. In both groups, three patients had a difference of $3 \mathrm{~mm}$ or more in anterior translation (KT-1000 at 
Table 3 One leg hop quotient between the operated and non-operated leg (\%) before anterior cruciate ligament reconstruction surgery and 12 and 24 months after. Values are mean (SD)

\begin{tabular}{llll}
\hline & Group I & Group II & p Value \\
\hline Before surgery & $87(11)$ & $76(15)$ & 0.0044 \\
12 months & $91(90)$ & $89(12)$ & 0.69 \\
24 months & $95(5)$ & $95(9)$ & 0.55 \\
\hline
\end{tabular}

Table 4 Anterior translation between the injured and healthy knee ( $m m ; K T-1000$ value at $89 \mathrm{~N}$ ) before anterior cruciate ligament reconstruction surgery and three, 12, and 24 months after. Values are mean (SD)

\begin{tabular}{lrll}
\hline & \multicolumn{1}{c}{ Group I } & Group II & p Value \\
\hline Before surgery & $2.8(0.9)$ & $3.3(1.6)$ & 0.19 \\
3 months & $-0.1(1.5)$ & $0.8(1.5)$ & 0.027 \\
12 months & $0.3(1.0)$ & $1.1(2.2)$ & 0.07 \\
24 months & $0.5(1.3)$ & $0.8(2.1)$ & 0.49 \\
\hline
\end{tabular}

Table 5 Anterior translation between the injured and healthy knee (mm; KT-1000 value at $135 \mathrm{~N}$ ) before anterior cruciate ligament reconstruction surgery and three, 12 , and 24 months after. Values are mean (SD)

\begin{tabular}{lrll}
\hline & \multicolumn{1}{c}{ Group I } & Group II & p Value \\
\hline Before surgery & $2.9(1.9)$ & $3.6(1.6)$ & 0.1 \\
3 months & $-0.3(1.2)$ & $0.8(1.7)$ & 0.0064 \\
12 months & $0.1(1.1)$ & $1.1(2.2)$ & 0.026 \\
24 months & $0.6(1.4)$ & $0.8(1.9)$ & 0.57 \\
\hline
\end{tabular}

Table 6 Patellofemoral pain score (maximum 20 points) before anterior cruciate ligament reconstruction surgery and three, 12, and 24 months after. Values are mean (SD)

\begin{tabular}{llll}
\hline & Group I & Group II & p Value \\
\hline Before surgery & $13(6)$ & $14(6)$ & 0.76 \\
3 months & $18(2)$ & $16(5)$ & 0.09 \\
12 months & $18(3)$ & $17(4)$ & 0.38 \\
24 months & $18(5)$ & $18(4)$ & 0.99 \\
\hline
\end{tabular}

$135 \mathrm{~N}$ ) between the operated and the healthy knee at the 24 month follow up. No difference was found between the groups at the 24 month follow up (tables 4 and 5).

PATELLOFEMORAL PAIN SCORE

After three months, both groups showed an improvement in the patellofemoral pain score over the preoperative value, and we found no signs of deterioration in this score after 12 and 24 months (table 6). No significant difference between the groups in terms of patellofemoral pain score was found before or after surgery.

IKDC SCORE

At the 24 month follow up, 12 patients were graded as normal (A), 16 as nearly normal (B), two as abnormal (C), and none as severely abnormal (D) in group I. In group II, 10 patients were graded as A, 15 as B, four as C, and none as $\mathrm{D}$ (table 7 ). Therefore the IKDC scores were similar for the two groups at the 24 month follow up.

COMPLICATIONS AND REPEAT OPERATIONS

One patient in group I experienced postoperative peroneal nerve palsy, probably because of an incorrectly positioned rehabilitation brace. The nerve palsy resolved completely within 12 months. One patient from each group received antibiotic treatment because of superficial infection and irritation in the operation wound,
Table 7 IKDC score at the 24 month follow up after anterior cruciate ligament reconstruction surgery

\begin{tabular}{lcc}
\hline & Group I & Group II \\
\hline A & 12 & 10 \\
B & 16 & 15 \\
C & 2 & 4 \\
D & 0 & 0
\end{tabular}

but cultures from knee joint aspirates were negative in both cases. Three patients from group I and one from group II suffered from postoperative haemarthrosis which required an arthrocentesis. Minor loss of extension $\left(5-10^{\circ}\right)$ was experienced by one patient in group I and by four patients in group II. One patient in group II had a significant loss of flexion $\left(15^{\circ}\right)$ at the two year follow up. Two patients in group I and four in group II were operated on again because of cartilage or meniscal injuries during the follow up period.

\section{Discussion}

The main findings in this study are that the "outside-in" and "all-inside" techniques produced identical functional results and knee stability after ACL reconstruction using bonepatellar tendon-bone grafts. The operation time was, however, longer using the "outsidein" technique and the mean sick leave was shorter. This difference did not, however, correlate with either subjective or objective outcome factors in the study. The shorter sick leave after the two incision technique may indicate less postoperative morbidity, which might make it the technique of choice because of the lower social cost associated with it.

One of the main reasons for failure of ACL reconstruction is probably incorrect placement of the femoral and tibial tunnels. ${ }^{9}$ To obtain an isometric placement of the graft, it is crucial to place the tunnels correctly in the tibia and femur. ${ }^{10-12}$ Concern has been voiced by some authors about the fact that the "all-inside" technique does not permit the same precision as the "outside-in" technique for placing the femoral tunnel, ${ }^{13}$ whereas others have pointed out that the "all-inside" technique using the "half-tunnel" drill guide could theoretically produce a more accurate placement. ${ }^{12}$

Harner et $a l^{7}$ found no significant differences between the two methods in their prospective study of 50 patients, in which more than half received allografts. A radiographic review showed that correct placement of the femoral tunnel was achieved with both methods.

Aglietti et $a l^{6}$ found no significant difference between the two methods in their prospective study. The median follow up was only 14 months. They concluded that there is a long learning curve for the "all-inside" technique but it may be preferable because of less postoperative pain and swelling.

Gröntvedt et $a l^{14}$ compared the placement reproducibility between the "all-inside" and the "outside-in" techniques and they found a statistically significant difference between the two methods along the proximal-distal coordinate, in which the "all-inside" technique led to a more proximal placement of the femoral tunnel than the "outside-in" technique. 
Another reason for the failure of the ACL reconstruction is insufficient fixation of the bone plugs with the interference screws. The pullout strength decreases if the fixation angle deviates by more than $20^{\circ}$ from the ideal parallel placement. ${ }^{15}$ With the "all-inside" technique, the placement of the femoral screw is more technically demanding than with the "outside-in" technique and the risk of a non-parallel screw placement resulting in graft failure is greater. As the femoral guide is passed through the tibial tunnel when the "all-inside" technique is used, the femoral tunnel is dependent on the correct placement of the tibial tunnel. When the "outside-in" technique is used, the femoral tunnel placement is, however, independent of the tibial tunnel. Using the "all-inside" technique, the two tunnels are ideally more in a straight line than with the "outside-in" technique and this could reduce the risk of wear related graft damage and ultimate graft failure. ${ }^{16}$ Sgaglione and Schwartz ${ }^{17}$ found no significant difference between the "outside-in" and "all-inside" techniques in a retrospective study. They noted screw divergence in $33 \%$ of the patients in the "all-inside" group compared with $14 \%$ in the "outside-in" group. This divergence did not, however, appear to have any effect on the final outcome.

In their retrospective study, Arciero et $a l^{18}$ found that both techniques produced a satisfactory final outcome in most patients, but severe intraoperative complications such as graft damage were more common in the "all-inside" group.

The results of the present study show that knee stability was restored in most patients and that knee function was satisfactory after both the "outside-in" and the "all-inside" technique in most patients. We now routinely use the one incision technique. These results are in accordance with previous reports. ${ }^{719-25}$ The rate of surgical complications was low in this study and no permanent disability was registered in our patients. There were significant improvements in patellofemoral pain scores in both groups after surgery.

The conclusion drawn from this study is that the "outside-in" and "all-inside" techniques are comparable in terms of functional results and stability at the 24 month follow up. The operation time was found to be somewhat shorter for the "all-inside" technique. The complication rate was low and no permanent disability was registered.

1 Eriksson E. Editorial. Knee Surg Sports Traumatol Arthrosc 1997;5:137.

2 Jones KG. Reconstruction of the anterior cruciate ligament: a technique using the central one-third of the patellar ligament. F Bone foint Surg [Am] 1963;45:825-931.

3 Dandy DJ. Historical overview of operations for anterior cruciate ligament rupture. Knee Surg Sports Traumatol cruciate ligament rupt

\section{Take home message}

Both the "outside-in" and "all-inside" arthroscopic methods can be used for anterior cruciate ligament reconstruction with equally good results. The method that feels most comfortable to the surgeon should therefore be chosen.
4 Järvinen $M$, Natri A, Lehto $M$, et al. Reconstruction of chronic anterior cruciate ligament insufficiency in athletes using a bone-patellar tendon-bone autograft. A two-year follow-up study. Int Orthop 1995;19:1-6.

5 Dandy DJ, Flanagan JP, Steenmeyer V. Arthroscopy and management of the ruptured anterior cruciate ligament. Clin Orthop 1982;167:43-9.

6 Aglietti P, Zaccherotti G, Menchetti PP, et al. A comparison of clinical and radiological parameters with two arthroscopic techniques for anterior cruciate ligament reconstruction. Knee Surg Sports Traumatol Arthrosc 1995;3:2-8.

7 Harner C, Marks, Fu F, Irrgang J, et al. Anterior cruciate ligament reconstruction: endoscopic versus two-incision technique. Arthroscopy 1994;10:502-12.

8 Santori N, Condello V, Maresca G, et al. Clinical and radiologic comparison of one- and two-incision technique for arthroscopic ACL reconstruction with patellar tendon. Orthopaedics International 1996;4:411-18.

8a Lysholm J, Gillquist J. Evaluation of knee ligament surgery results with special emphasis on the use of a scoring scale. Am $\mathcal{F}$ Sports Med 1982;10:150-4.

$8 \mathrm{~b}$ Tegner Y, Lysholm J. Rating systems in evaluation of the knee ligament injuries. Clin Orthop 1985;198:43-9.

8c Hefti F, Müller W, Jakob RP, et al. Evaluation of knee ligament injuries with the IKDC form. Knee Surg Sports Tranmatol Arthrosc 1993;1:226-34.

9 Good L, Odensten M, Gillquist J. Sagittal knee stability after anterior cruciate ligament reconstruction with a patellar tendon strip. A two-year follow-up study. $A m \mathcal{F}$ Sports Med 1994;22:518-23.

10 Penner DA, Daniel DM, Wood P, et al. An in vitro study of anterior cruciate ligament graft placement and isometry. Am $\mathcal{F}$ Sports Med 1988;16:238-43.

11 Romano VM, Graf BK, Keene JS, et al. Anterior cruciate ligament reconstruction. The effect of tibial tunnel placement on range of motion. Am 7 Sports Med 1993;21:415-18.

12 Schutzer SF, Christen S, Jakob RP. Further observations on the isometricity of the anterior cruciate ligament. An anatomical study using a $6-\mathrm{mm}$ diameter replacement. Clin Orthop 1989;242:247-55.

13 Lemos M, Albert J, Simon T, et al. Radiographic analysis of femoral interference screw placement during ACL reconstruction: endoscopic versus open technique. Arthroscopy 1993;9:154-8.

14 Gröntvedt T, Pena F, Engebretsen L. Accuracy of femoral tunnel placement and resulting graft force using one- or two-incision drill guides. A cadaver study on ten paired knees. Arthroscopy 1996;12:187-92.

15 Jomha NM, Raso VJ, Leung P. Effect of varying angles on the pullout strength of interference screw fixation. Arthroscopy 1993;9:580-3.

16 Graf BK, Henry J, Rothenberg M, et al. Anterior cruciate ligament reconstruction with patellar tendon. An ex vivo study of wear-related damage and failure at the femoral tunnel. Am f Sports Med 1994;22:131-5.

17 Sgaglione NA, Schwartz RE. Arthroscopically assisted reconstruction of the anterior cruciate ligament: initial clinical experience and minimal 2-year follow-up comparing endoscopic transtibial and two-incision techniques. Arthroscopy 1997;13:156-65.

18 Arciero RA, Scoville CR, Snyder RJ, et al. Single versus twoincision arthroscopic anterior cruciate ligament reconstruction. Arthroscopy 1996;4:462-9.

19 Aglietti P, Buzzi R, D'Andria S, et al. Arthroscopic anterior cruciate ligament reconstruction with patellar tendon. Arthroscopy 1995;8:510-16.

20 Bach BRJ, Jones GT, Sweet FA, et al. Arthroscopy-assisted anterior cruciate ligament reconstruction using patellar tendon substitution. Two- to four-year follow-up results. Am F Sports Med 1994;22:758-67.

21 Buss DD, Warren RF, Wickiewicz TL, et al. Arthroscopically assisted reconstruction of the anterior cruciate ligament with use of autogenous patellar-ligament grafts. Results after twenty-four to forty-two months. $\mathcal{F}$ Bone foint Surg $[A m]$ 1993;75:1346-55.

22 Harilainen A, Sandelin J, Osterman K, et al. Prospective preoperative evaluation of anterior cruciate ligament instability of the knee joint and results of reconstruction with patellar ligament. Clin Orthop 1993;297:17-22.

23 Howe JG, Johnson RJ, Kaplan MJ, et al. Anterior cruciate ligament reconstruction using quadriceps patellar tendon graft. Part I. Long-term follow-up. Am $\mathcal{F}$ Sports Med 1991; 19:447-57.

24 O'Brien SJ, Warren RF, Pavlov H, et al. Reconstruction of the chronically insufficient anterior cruciate ligament with the central third of the patellar ligament. F Bone foint Surg 1991;73:278-86.

25 Paulos LE, Cherf J, Rosenberg TD, et al. Anterior cruciate ligament reconstruction with autografts. Clin Sports Med 1991;10:463-8. 